\title{
DLCQ and Plane Wave Matrix Big Bang Models
}

\author{
Matthias Blau ${ }^{a}$ and Martin O'Loughlin ${ }^{b}$ \\ ${ }^{a}$ Institut de Physique, Université de Neuchâtel, Breguet 1, Neuchâtel, Switzerland \\ ${ }^{b}$ University of Nova Gorica, Vipavska 13, 5000 Nova Gorica, Slovenia
}

\begin{abstract}
We study the generalisations of the Craps-Sethi-Verlinde matrix big bang model to curved, in particular plane wave, space-times, beginning with a careful discussion of the DLCQ procedure. Singular homogeneous plane waves are ideal toy-models of realistic space-time singularities since they have been shown to arise universally as their Penrose limits, and we emphasise the role played by the symmetries of these plane waves in implementing the flat space Seiberg-Sen DLCQ prescription for these curved backgrounds. We then analyse various aspects of the resulting matrix string Yang-Mills theories, such as the relation between strong coupling space-time singularities and world-sheet tachyonic mass terms. In order to have concrete examples at hand, in an appendix we determine and analyse the IIA singular homogeneous plane wave - null dilaton backgrounds.
\end{abstract}




\section{Contents}

1 Introduction $\quad 3$

2 DLCQ and Singular Homogeneous Plane Wave Backgrounds $\quad 5$

2.1 Quick Recap of the Seiberg-Sen Argument . . . . . . . . . . . . . . . . . . . . 5

2.2 Adapting the Seiberg-Sen Argument to the CSV Setting . . . . . . . . . . . . . . . 7

2.3 Extending the Seiberg-Sen-CSV DLCQ to Plane Wave Backgrounds: the Privileged Role of Singular Homogeneous Plane Waves . . . . . . . . . . . . . . . . . 9

$2.4 \quad$ 9/11 Flip, DBI Expansion, and Decoupling _ . . . . . . . . . . . . . . . . . . 13

3 Some Basic Properties of the Matrix String Action for SHPWs 15

3.1 The Plane Wave Matrix String Action in Rosen Coordinates . . . . . . . . . . . . . . . . 15

3.2 Rosen vs Brinkmann Form of the Matrix String Action . . . . . . . . . . . . . . . . . . 17

3.3 Absorbing the Coupling Constant into the Worldsheet Metric . . . . . . . . . . . . . 19

3.4 Tachyons and Strong String Coupling Singularities . . . . . . . . . . . . . . . . . . . . 20

$\begin{array}{lr}\text { A Plane Wave Geometry: Synopsis } & 21\end{array}$

B A Class of Plane Wave - Null Dilaton Big Bang Backgrounds 23

B.1 M $\rightarrow$ IIA Reduction of Singular Homogeneous Plane Wave Backgrounds . . . . . . . . . . 23

B.2 Equations of Motion . . . . . . . . . . . . . . . . . . . . . . . . . . . 24

B.3 Singularity Structure and Behaviour of the Dilaton at the Singularity . . . . . . . . . 25

$\begin{array}{lr}\text { References } & 26\end{array}$ 


\section{INTRODUCTION}

One of the main aims of string theory, as a theory of quantum gravity, is to elucidate the nature and fate of space-time singularities. String propagation in static space-times, such as time-independent orbifold singularity backgrounds, is reasonably well understood. However, comparatively little is known about string theory in non-trivial time-dependent (and possibly singular) space-time backgrounds, the time-dependence giving rise to rather basic problems in the very formulation of string theory in such backgrounds, and the singularities making questionable the validity or reliability of a perturbative approach to the problem. It is thus natural to appeal to more modern non-perturbative formulations of string theory to gain some insight into these issues. An excellent summary of recent research along these lines, e.g. via the AdS/CFT correspondence or tachyon condensation, can be found in [1].

One can also try to use non-perturbative matrix theory formulations of M-theory $[2,3]$ or string theory $[4,5]$ to address the fate of singularities. What had hampered progress along these lines is the fact that these theories are quite complicated in general (even weakly) curved backgrounds $[6,7]$. However, it has recently been pointed out by Craps, Sethi and Verlinde (CSV) [8] that one can find an explicit matrix string description of a particular time-dependent IIA background, given in the string frame by a flat metric with a linear null dilaton. This leads to a metric with a null singularity either in the Einstein frame or upon lifting this configuration to Mtheory. The central observation of [8] is that the dual matrix string gauge theory description of string theory in this background is well-defined and weakly coupled close to the singularity. In this regime the non-Abelian nature of the matrix-string coordinates cannot be neglected and one thus tentatively arrives at a picture where space-time geometry becomes non-commutative near a singularity, while the emergence of a classical space-time at large distances from the singularity has been confirmed by a 1-loop calculation $[9,10]$. Subsequently, the CSV model has been extended and generalised in various ways, e.g. to null brane backgrounds $[11,12,13]$ and certain plane wave metrics $[14,15,16,17]$.

Here we will carefully revisit the extension of the CSV model to singular plane wave backgrounds, not only because such geometries exhibit a perfect combination of simplicity in construction together with interesting non-trivial features such as time-dependence and a singularity, but also because the extension of the Seiberg-Sen DLCQ procedure [18, 19, 20] to curved backgrounds requires some care, and we feel that previous treatements of this issue have not always been wholly satisfactory.

Moreover, as we will now argue, in the context of the matrix string theory description of spacetime singularities there is a privileged class of IIA plane wave backgrounds, the homogeneous or scale-invariant singular plane waves $[21,22]$ with a null dilaton. Thanks to the special properties of these metrics they provide us with a natural and interesting generalisation of the CSV background that also permits an almost literal implementation of the generally accepted flat space Seiberg-Sen procedure in these curved backgrounds.

We begin with the simple observation that the CSV IIA background (B.2), namely Minkowski 
space with a linear dilaton, lifts to the 11-dimensional metric

$$
d s_{11}^{2}=-2 d u d v+u \delta_{i j} d y^{i} d y^{j}+u^{-2}(d y)^{2} .
$$

This exhibits the CSV background, first of all, as a special case of an 11-dimensional plane wave, whose general form in Rosen coordinates is $d s_{11}^{2}=-2 d u d v+G_{\mu \nu}(u) d y^{\mu} d y^{\nu}$. Secondly, and more specifically, the CSV background falls into the special class of plane wave metrics with the power-law behaviour

$$
d s_{11}^{2}=-2 d u d v+\sum_{i} u^{2 n_{i}}\left(d y^{i}\right)^{2}+u^{2 b}(d y)^{2} .
$$

It is precisely this class of metrics that was argued to provide an ideal class of models of realistic space-time singularities, because these singular scale-invariant homogeneous plane waves [22] were shown in $[23,24,25]$ to arise generically as the Penrose limits of space-time singularities of power-law type [26]. ${ }^{1}$ Thus these metrics are not just toy-models but true approximations (in the sense of the Penrose-Fermi expansion [27, 28]) of space-time singularities and, as such, provide a physically well-motivated generalisation of the CSV background.

We thus need to understand how to extend the DLCQ construction $[18,19]$ of matrix string theory, briefly reviewed in section 2.1 following [20], to these curved backgrounds. To that end, we first carefully rephrase the procedure adopted by CSV in the Seiberg-Sen framework (section 2.2). Having clarified what are precisely the steps involved, in particular a null rotation (aligning the almost null with a spacelike circle), a boost of the energies, and a subsequent overall rescaling of the length scales of the theory, we then show that, remarkably, the singular homogeneous plane waves are precisely such that these operations can be implemented via isometries and homotheties of the metric (section 2.3). We also resolve some ambiguities regarding the order in which these isometries, scalings and the duality transformations implementing the $9 / 11$ flip [5] of matrix string theory are to be performed. This then allows us, as in [8], to deduce the matrix string theory action from the expansion of the D-string DBI action (section 2.4) and for its bosonic part we obtain, schematically (for the precise expression see (3.2)),

$$
S \sim \int d^{2} \sigma \operatorname{Tr}\left(\mathrm{e}^{2 \phi(\tau)} F_{\alpha \beta} F^{\alpha \beta}+g_{i j}(\tau) D_{\alpha} X^{i} D^{\alpha} X^{j}+\mathrm{e}^{-2 \phi(\tau)} g_{i k}(\tau) g_{j l}(\tau)\left[X^{i}, X^{j}\right]\left[X^{k}, X^{l}\right]\right)
$$

where the $g_{i j}$ are the components of the IIA Rosen coordinate plane wave metric, and $\phi$ is the null dilaton. These actions, which are the natural plane wave counterparts of ordinary YangMills theory, have appeared before in this context, e.g. in [14, 15, 17], but both in our derivation of this DLCQ matrix string action and in the analysis of the decoupling conditions (justifying the truncation to this world-sheet Yang-Mills-like theory) our treatment differs significantly from that of previous publications.

Having obtained this action, we then analyse some of its properties. We comment on the fermionic terms in the action in section 3.1. In section 3.2 we show that the space-time coordinate transformation from Rosen to Brinkmann coordinates results in a rather non-obvious equivalence between two apparently very different matrix string Yang-Mills theories (namely the above

\footnotetext{
${ }^{1}$ This is a rather mild condition which essentially says that the singularity should not be non-analytic in some suitably chosen coordinates.
} 
action, with time-dependent couplings for the scalar fields on the one hand, and the Brinkmann action (3.9) with time-independent couplings but time-dependent mass terms instead). This illustrates that the above action correctly captures the target space-time geometry. The brief discussion of this equivalence presented here will be supplemented by a more detailed discussion in [29], where we also extend it to 3-algebra actions such as those that appear in the BLG multiple M2-brane actions [30, 31, 32],

In section 3.3 we investigate the possibility to absorb the time-dependent couplings into the world-sheet metric, as in [8], finding one special case where one ends up with a $(1+1)$ de Sitter world-sheet, and in section 3.4 we highlight the usefulness of the (more space-time covariant) Brinkmann representation of the action by showing how the space-time nature of the singularity (whether it is strongly or weakly coupled) is reflected in the mass terms of the world-sheet theory (strong coupling requiring at least one 'tachyonic' scalar).

We briefly summarise some useful facts about the geometry of plane waves in appendix A, and in appendix B we classify and analyse the plane wave - null dilaton backgrounds that arise upon reduction from the $11 \mathrm{~d}$ plane wave power-law metrics (1.2).

One subject we do not address here is that of classical solutions in these models, such as fuzzy spheres, and the possible role that they may play in understanding the evolution away from/towards the null singularity. These have been studied before in this context, e.g. in $[16,15]$, and some interesting new work in this direction will appear in [33].

\section{DlCQ and Singular Homogeneous Plane Wave Backgrounds}

\subsection{Quick Recap of the Seiberg-Sen Argument}

In the Seiberg-Sen argument $[18,19]$ (as well as in its CSV variant $[8,10]$ we will discuss below) a central role is played by the transformation which relates the compactification on a (vanishingly) small spacelike circle with radius $R_{s} \rightarrow 0$ to the DLCQ compactification on a null circle with fixed radius $R$. Concretely, one considers the metric

$$
d s^{2}=-2 d y^{+} d y^{-}+\ldots=-\left(d y^{0}\right)^{2}+\left(d y^{9}\right)^{2}+\ldots
$$

where $y^{ \pm}=\left(y^{0} \pm y^{9}\right) / \sqrt{2}$. In order to realise the lightlike identification $y^{-} \sim y^{-} \pm 2 \pi R$ as a limit of standard spacelike compactifications, one considers the boost

$$
x^{ \pm} \equiv y^{\prime \pm}=\mathrm{e}^{ \pm \beta} y^{ \pm}
$$

and requires that in the boosted/primed coordinates the identification is

$$
x^{9} \sim x^{9}+2 \pi R_{s} \quad x^{0} \sim x^{0} .
$$

A convenient choice is e $\mathrm{e}^{\beta}=\sqrt{2} \frac{R}{R_{s}}$, corresponding to the simple identification

$$
y^{+} \sim y^{+}+\pi R_{s}^{2} / R \quad y^{-} \sim y^{-}-2 \pi R
$$


of the unboosted lightcone coordinates $y^{ \pm}$(with this choice of $\beta$ the periodicity of $y^{-}$is fixed, i.e. independent of $R_{s}$ ). Since the lightcone coordinates transform under a boost as in (2.2), the momenta transform as $p_{ \pm}^{\prime}=\mathrm{e}^{\mp \beta} p_{ \pm}$. In particular, a state with $N$ units of momentum in the compact $x^{9}$-direction, $p_{9}^{\prime}=N / R_{s}$, leads in the limit $R_{s} \rightarrow 0$ to a state with $N$ units of lightcone momentum

$$
p^{+} \equiv-p_{-}=\frac{N}{R}
$$

since

$$
\frac{N}{R_{s}}=p_{9}^{\prime}=\frac{1}{\sqrt{2}}\left(p_{+}^{\prime}-p_{-}^{\prime}\right)=\frac{1}{\sqrt{2}}\left(\mathrm{e}^{-\beta} p_{+}-\mathrm{e}^{\beta} p_{-}\right) \stackrel{R_{s} \rightarrow 0}{\longrightarrow}-\frac{R}{R_{s}} p_{-}
$$

Now, following [20], one defines the DLCQ Hamiltonian $H_{N}^{D L C Q}(m, R)$ (in a sector with $N$ units of lightcone momentum and characterised by, say, a mass scale $m$ ) as the limit

$$
H_{N}^{D L C Q}(m, R):=\lim _{R_{s} \rightarrow 0} i \partial_{y^{+}}
$$

with $m$ and $R$ fixed, since in this limit the spacelike identification (2.3) becomes the lightlike identification $y^{-} \sim y^{-}+2 \pi R$. In terms of the boosted coordinates $x^{\mu}=y^{\prime \mu}$ one has

$$
E_{l c} \equiv i \partial_{y^{+}}=\frac{1}{\sqrt{2}} \mathrm{e}^{\beta}\left(i \partial_{x^{0}}+i \partial_{x^{9}}\right)=\frac{R}{R_{s}}\left(E^{\prime}-p_{9}^{\prime}\right)
$$

The term in brackets on the rhs is the total energy of the system minus the background energy $p_{9}^{\prime}=N / R_{s}$, Sen's $[19,20] \mathrm{KK}$ Hamiltonian $H_{N}\left(m, R_{s}\right):=E^{\prime}-p_{9}^{\prime}$. In the present context, it is also useful to think of this linear combination in the limit $p_{9}^{\prime}=N / R_{s} \rightarrow \infty$ as the non-relativistic infinite momentum frame Hamiltonian

$$
E^{\prime}=\sqrt{\left(p_{9}^{\prime}\right)^{2}+\vec{p}^{\prime 2}+m^{2}} \Rightarrow E^{\prime}-p_{9}^{\prime}=\frac{\vec{p}^{\prime 2}+m^{2}}{2 p_{9}^{\prime}}+\mathcal{O}\left(\left(p_{9}^{\prime}\right)^{-3}\right)
$$

Thus one has

$$
H_{N}^{D L C Q}(m, R)=\lim _{R_{s} \rightarrow 0} \frac{R}{R_{s}} H_{N}\left(m, R_{s}\right)
$$

In order to identify the rhs, and eliminate the singular prefactor, one now makes the observation that, on purely dimensional grounds, if one rescales all mass scales by a factor $\lambda$, and all length scales by a factor $\lambda^{-1}$, then the Hamiltonian will also scale as $\lambda$. Thus, with $\lambda=R / R_{s}$ one has

$$
\frac{R}{R_{s}} H_{N}\left(m, R_{s}\right)=H_{N}\left(\frac{R}{R_{s}} m, \frac{R_{s}^{2}}{R}\right) \equiv H_{N}\left(\hat{m}, \hat{R}_{s}\right),
$$

and one has now concretely realised the DLCQ Hamiltonian as the limit

$$
H_{N}^{D L C Q}(m, R)=\lim _{\hat{R}_{s} \rightarrow 0} H_{N}\left(\hat{m}, \hat{R}_{s}\right)
$$

of standard KK Hamiltonians of a family of new theories with mass scale $\hat{m}=\left(R / R_{s}\right) m$ and spatial radius $\hat{R}_{s}=R_{s}^{2} / R$, with $m$ and $R$ held fixed. ${ }^{2}$

This much is completely general, and pure kinematics. One can now apply this to $M$-theory with $m=m_{p}$ the Planck mass and $R_{s}=R_{11}$. In terms of the scaled IIA parameters $\hat{g}_{s}$ and

\footnotetext{
${ }^{2}$ Note that, while in terms of $R_{s}$ one has $R_{s} \hat{m}=R m$ (this really is just a change of scale, the dimensionless quantity $R m$ being kept fixed), in terms of $\hat{R}_{s}$ one has the Seiberg relation $\hat{R}_{s} \hat{m}^{2}=R m^{2}$ [18].
} 
$\hat{\ell}_{s}$ (string coupling and string length), this is then precisely the DKPS [34] D0-brane weakly coupled $\left(\hat{g}_{s} \rightarrow 0\right)$ field theory $\left(\hat{\ell}_{s} \rightarrow 0\right)$ limit, in which the Hamiltonian reduces to YM quantum mechanics with the finite YM coupling $g_{Y M}^{2}=\hat{g}_{s} \hat{\ell}_{s}^{-3}$, leading to the BFSS matrix theory [2].

One can equally well apply this prescription to IIA string theory $[4,5,35]$. Thus, starting off with a IIA theory with string scale $m_{s}$ and null circle with radius $R$, this is described by the limit of a $\widehat{I I A}$-theory with string scale $\hat{m}_{s}=\left(R / R_{s}\right) m_{s}$ and radius $\hat{R}_{s}=R_{s}^{2} / R$, and with the same (dimensionless) coupling constant $\hat{g}_{s}=g_{s}$. To identify this theory, one can lift it to 11dimensions. Then one sees that this theory is described by the above YM matrix theory with an additional transverse circle, bettter described by $(1+1)$-dimensional YM theory on the dual cylinder with constant YM coupling constant $g_{Y M}^{2} \sim\left(R / g_{s} \ell_{s}^{2}\right)^{2}$ and constant radius $R_{D 1}=\ell_{s}^{2} / R$ on which the T-dual D1-branes are wrapped. This is the matrix string DLCQ description of IIA string theory.

\subsection{Adapting the Seiberg-Sen Argument to the CSV Setting}

In $[8,10]$, a variant of the Seiberg-Sen argument was introduced, in which the lightlike compactification is related to a limit of spacelike compactifications along a direction transverse to the lightcone. The motivation for this was the fact that in the CSV model one has a null linear dilaton, which is not compatible with the periodic identification of $y^{+}$in (2.4). In this section, in order to prepare the ground for the generalisation to curved backgrounds, we will explain the precise relationship between the procedure adopted by CSV and the Seiberg-Sen argument of the previous section.

The point of departure this time is a metric of the form

$$
d s^{2}=-2 d y^{+} d y^{-}+\left(d y^{1}\right)^{2}+\ldots,
$$

where we seek a Lorentz tranformation such that an almost null identification in this background is mapped to the manifestly spatial identification $\tilde{x}^{1} \sim \tilde{x}^{1}+2 \pi R_{s}$. If one does (for the time being) not touch $y^{+}$, this leaves null rotations which have the general form

$$
y^{-}=\tilde{x}^{-}+\alpha \tilde{x}^{1}+\left(\alpha^{2} / 2\right) \tilde{x}^{+} \quad y^{1}=\tilde{x}^{1}+\alpha \tilde{x}^{+} .
$$

The identification then becomes

$$
y^{1} \sim y^{1}+2 \pi R_{s} \quad y^{-} \sim y^{-}+2 \pi \alpha R_{s}
$$

Thus the obvious choice for $\alpha$ (leading, as in section 2.1, to an $R_{s}$-independent periodicity of $y^{-}$), is $\alpha= \pm R / R_{s}$. Once again, a state with $N$ units of momentum in the compact direction, $\tilde{p}_{1}=\mp N / R_{s}$, is mapped in the limit $R_{s} \rightarrow 0$ to a state with $N$ units of lightcone momentum $p^{+}=N / R$, since

$$
\tilde{p}_{1}=p_{1}+\alpha p_{-}=p_{1} \pm \frac{R}{R_{s}} p_{-} \stackrel{R_{s} \rightarrow 0}{\longrightarrow} \pm \frac{R}{R_{s}} p_{-} .
$$

Therefore, we can again define the DLCQ Hamiltonian as the limit

$$
H_{N}^{D L C Q}(m, R):=\lim _{R_{s} \rightarrow 0} i \partial_{y^{+}}
$$


this time with the almost null identification (2.15), and the aim is now to rewrite this as a well-defined limit of standard Hamiltonians. Here it is important to note that, while in the Seiberg-Sen procedure the boosting of the energies, as in (2.8), was an automatic consequence of aligning an almost lightlike with a manifestly spacelike direction, here this is not the case. Rather, under the null rotation, which achieves this alignment all by itself, the lightcone energy transforms as

$$
\tilde{E}_{l c}=E_{l c}-\alpha p_{1}-\left(\alpha^{2} / 2\right) p_{-}
$$

and is thus not becoming small. Thus one can anticipate that this boost must still be performed seperately in order to arrive at the vanishing energies that permit a decoupling limit argument. Moreover, the Seiberg-Sen boost had the added bonus that it automatically led to the appropriate background subtracted KK Hamiltonian $H_{N}=E^{\prime}-p_{9}^{\prime}$. Using the CSV prescription, this is not automatically the case, the background momentum arising from the $x^{1}$-direction, which is not part of the lightcone directions. However, we will see that with a judicious choice of null rotation and boost parameters we will once again be able to relate the DLCQ Hamiltonian to the relevant KK Hamiltonian, namely $E^{\prime}-p_{1}^{\prime}$.

To make this more explicit, we go to the adapted null-rotated coordinate system $\tilde{x}^{\mu}(2.14)$, and also perform a further boost isometry, with parameter $\gamma>0$, to the coordinates $y^{\prime \mu}=x^{\mu}$, with

$$
x^{ \pm}=\gamma^{ \pm 1} \tilde{x}^{ \pm}
$$

Then one has the relation

$$
i \partial_{y^{+}}=\gamma i \partial_{x^{+}}-\alpha i \partial_{x^{1}}+\gamma^{-1} \alpha^{2} / 2 i \partial_{x^{-}} \quad \Leftrightarrow \quad E_{l c}=\gamma\left(E_{l c}^{\prime}+(\alpha / \gamma) p_{1}^{\prime}-\left(\alpha^{2} / 2 \gamma^{2}\right) p_{-}^{\prime}\right)
$$

To unravel this, let us write the lightcone energies and momenta in terms of the ordinary energy $E^{\prime}=i \partial_{x^{0}}$ and $p_{9}^{\prime}=-i \partial_{x^{9}}$,

$$
E_{l c}=\frac{\gamma}{\sqrt{2}}\left(\left(1+\alpha^{2} / 2 \gamma^{2}\right) E^{\prime}-\left(1-\alpha^{2} / 2 \gamma^{2}\right) p_{9}^{\prime}+(\sqrt{2} \alpha / \gamma) p_{1}^{\prime}\right)
$$

We see that we can eliminate the annoying $p_{9}^{\prime}$ from this expression by choosing $\alpha$ and $\gamma$ such that $\alpha^{2} / 2 \gamma^{2}=1$. This also has the consequence that $p_{1}^{\prime}$ appears with the same coefficient as $E^{\prime}$. Specifically, choosing

$$
\alpha=-\frac{R}{R_{s}} \quad \gamma=\frac{R}{\sqrt{2} R_{s}}
$$

one finds the simple result

$$
E_{l c}=\frac{\gamma}{\sqrt{2}}\left(2 E^{\prime}-2 p_{1}^{\prime}\right)=\frac{R}{R_{s}}\left(E^{\prime}-p_{1}^{\prime}\right)
$$

which is the precise analogue of the expression (2.8) that appears in the argument based on the Seiberg boost. The expression in brackets is Sen's KK Hamiltonian, and thus one can now repeat verbatim the arguments of the previous section to deduce that

$$
H_{N}^{D L C Q}(m, R)=\lim _{R_{s} \rightarrow 0} \frac{R}{R_{s}} H_{N}\left(m, R_{s}\right)=\lim _{\hat{R}_{s} \rightarrow 0} H_{N}\left(\hat{m}, \hat{R}_{s}\right) .
$$

The change of scale involved in the last equality means that the relation (2.23) between the lightcone energy and the boosted energy becomes the statement that the original lightcone 
energy is now equal to the background subtracted energy in the final (YM-like in the string context) rescaled theory,

$$
E_{l c}=\hat{E}-\hat{p}_{1} .
$$

It is convenient, not only for book-keeping purposes, to concretely implement this change of scale by the scaling

$$
\hat{x}^{\mu}=\frac{R_{s}}{R} x^{\mu} \quad d \hat{s}^{2}=\left(\frac{R_{s}}{R}\right)^{2} d s^{2} .
$$

of the coordinates and the metric. In particular, the new energies and momenta are now related to the old ones by $\hat{E}=\left(R / R_{s}\right) E^{\prime}$ etc., leading to $(2.25)$. Moreover, the concomitant rescaling of the metric has precisely the effect that other length scales in the problem, like transverse radii, are also automatically rescaled appropriately, i.e. a circle of proper radius $\rho$ with respect to the metric $d s^{2}$ has proper radius $\hat{\rho}=\left(R_{s} / R\right) \rho$ with respect to the metric $d \hat{s}^{2}$.

We should note here that, while the above choice of parameters (2.22) leads to a nice cancellation among various terms, and thus to the simple final result (2.25), there is considerable leeway in the choice of parameters if one is only interested in some gauge-fixed energy fluctuations. In particular if, in the string context and as in [8], one gauge fixes $x^{+}$and $x^{1}$, this amounts to setting the fluctuations $\delta \hat{p}_{-}$and $\delta \hat{p}_{1}$ to zero. This implies, in particular, that $\delta \hat{E}_{l c} \sim \delta \hat{E}$, so that at the level of the gauge-fixed fluctuations (2.21) leads to

$$
\delta E_{l c} \sim \delta \hat{E} \quad \forall \gamma \sim \alpha= \pm \frac{R}{R_{s}}
$$

(with a finite proportionality factor), which thus also gives a direct relationship between the lightcone and YM (fluctuation) energies. In particular, the choice adopted by CSV is

$$
\alpha=\gamma=\frac{R}{R_{s}},
$$

leading to the (apparently less attractive) result

$$
E_{l c}=\left(\hat{E}_{l c}+\hat{p}_{1}-\frac{1}{2} \hat{p}_{-}\right)
$$

which, however, reduces to (2.27) at the level of fluctuations. This is good enough. Below, when discussing the generalisation of this procedure to curved backgrounds, we will similarly make use of this freedom (and adopt the CSV choice (2.28)) to define an appropriate fluctuation Hamiltonian since, in general, in any case there will be no choice of constant parameters $\alpha, \gamma$ that gives on the nose the appropriate curved space analogue $(2.45)$ of $(2.9,2.25)$.

\subsection{Extending the Seiberg-Sen-CSV DLCQ to Plane Wave Backgrounds: the Privileged Role of Singular Homogeneous Plane Waves}

We will now discuss the generalisation of the CSV derivation of matrix string theory to curved (in particular plane wave) backgrounds. To that end, let us first take stock of the steps involved in the CSV procedure. As we saw above, in the case of a flat background (possibly supplemented by a null dilaton [8]), the derivation can be concretely implemented by the following three steps: 
1. As a first step, one performs the purely kinematical operation of passing to adapted coordinates by a coordinate transformation that aligns the almost null with a small space-like circle. In the case of the flat metric this is accomplished by a null rotation Lorentz isometry.

2. Next one performs a dynamical transformation (acting non-trivially on the lightcone time coordinate $y^{+}$) which has the effect of rescaling the energies. In the case of the flat metric this is accomplished by a boost Lorentz isometry.

3. Finally, this boost is accompanied by a rescaling of the coordinates (and the metric), implementing the Seiberg-Sen change of mass/length scales. In the case of the flat metric this is accomplished by a uniform scaling of the Minkowski coordinates, which is a homothety (constant conformal rescaling) of the metric.

We will now show that this prescription can be implemented almost literally for a special class of plane wave metrics (in the above, replace "flat" by "singular homogeneous plane wave", "Minkowski" by "Brinkmann", and eliminate the word "Lorentz").

To set the stage, we first consider a general plane wave metric, which in Rosen coordinates takes the form

$$
d s^{2}=-2 d y^{+} d y^{-}+g_{i j}\left(y^{+}\right) d y^{i} d y^{j} .
$$

Note that this metric has manifest translational isometries in the lightcone $y^{-}$- and transverse $y^{i}$-directions, and we thus begin with the almost null identification

$$
\left(y^{+}, y^{-}, y^{1}, y^{m}\right) \sim\left(y^{+}, y^{-}+2 \pi R, y^{1}+2 \pi \epsilon R, y^{m}\right) .
$$

where we have set $R_{s}=\epsilon R$. As before, we would like to perform a coordinate transformation (and ideally an isometry) $y^{\mu} \rightarrow \tilde{x}^{\mu}$, in terms of which the above identification simply reads

$$
\tilde{x}^{1} \sim \tilde{x}^{1}+2 \pi \epsilon R .
$$

Now in addition to the manifest transverse translational isometries, any plane wave metric (2.30) has dual hidden translational symmetries generated by the transverse Killing vectors $P^{(i)}$ (A.4). In particular, $P^{(1)}$ generates the transformation (null rotation)

$$
\left(y^{+}, y^{-}, y^{1}, y^{m}\right)=\left(\tilde{x}^{+}, \tilde{x}^{-}+\alpha \tilde{x}^{1}+\alpha^{2} h^{11}\left(\tilde{x}^{+}\right) / 2, \tilde{x}^{1}+\alpha h^{11}\left(\tilde{x}^{+}\right), \tilde{x}^{m}+\alpha h^{1 m}\left(\tilde{x}^{+}\right)\right),
$$

(where $h^{i k}\left(y^{+}\right)=\int^{y^{+}} d u g^{i k}(u)$ ), which indeed accomplishes (2.32) for the choice $\alpha=\epsilon^{-1}$.

Thus step 1 can be implemented via isometries for any plane wave metric. Step 2 requires an isometry involving transformations of $y^{+}$. Generic plane waves do not possess any such isometries. However, there are precisely two classes of plane waves with such an extra isometry [22]. In one, this isometry involves a shift in $y^{+}$, in the other the isometry is realised not by a shift but by a scaling of $y^{+}$(accompanied by some transformation of the other coordinates). In the present context, it is evidently this latter class of singular homogeneous plane waves (SHPWs) that we are interested in. The prototypical example of such SHPWs are plane wave metrics with a power-law behaviour in Rosen coordinates,

$$
d s^{2}=-2 d y^{+} d y^{-}+\sum_{i}\left(y^{+}\right)^{2 m_{i}}\left(d y^{i}\right)^{2} .
$$


This isometry is more manifest in Brinkmann coordinates, in which the above metric takes the form

$$
d s^{2}=-2 d z^{+} d z^{-}+\sum_{a} m_{a}\left(m_{a}-1\right)\left(z^{a}\right)^{2} \frac{\left(d z^{+}\right)^{2}}{\left(z^{+}\right)^{2}}+\sum_{a}\left(d z^{a}\right)^{2} .
$$

This metric clearly possesses the isometry is $\left(z^{+}, z^{-}\right) \rightarrow\left(\lambda z^{+}, \lambda^{-1} z^{-}\right)$and, translated back to Rosen coordinates, this isometry is given by $\left(y^{+}, y^{-}, y^{i}\right) \rightarrow\left(\lambda y^{+}, \lambda^{-1} y^{-}, \lambda^{-m_{i}} y^{i}\right)$. In particular, the Lorentz boost isometry (2.19) can be generalised to the isometry

$$
\left(\tilde{x}^{+}, \tilde{x}^{-}, \tilde{x}^{i}\right)=\left(\gamma^{-1} x^{+}, \gamma x^{-}, \gamma^{m_{i}} x^{i}\right),
$$

and we will eventually choose $\gamma=\alpha=\epsilon^{-1}$, in accordance with (2.28).

Now let us consider Step 3, the scaling. First of all we note that any plane wave

$$
d s^{2}=-2 d z^{+} d z^{-}+A_{a b}\left(z^{+}\right) z^{a} z^{b}\left(d z^{+}\right)^{2}+\left(d z^{a}\right)^{2}=-2 d x^{+} d x^{-}+g_{i j}\left(x^{+}\right) d x^{i} d x^{j}
$$

has the homothety (conformal isometry with a constant factor)

$$
\left(z^{+}, z^{-}, z^{a}\right) \rightarrow\left(z^{+}, \lambda^{2} z^{-}, \lambda z^{a}\right) \quad \Leftrightarrow \quad\left(x^{+}, x^{-}, x^{i}\right) \rightarrow\left(x^{+}, \lambda^{2} x^{-}, \lambda x^{i}\right)
$$

under which $d s^{2} \rightarrow \lambda^{2} d s^{2}$. Even though this scales the metric, it does not uniformly rescale the energies / length scales of the theory as e.g. $x^{+}$does not scale. However, precisely when $A_{a b}\left(z^{+}\right) \sim\left(z^{+}\right)^{-2}$, as in (2.35), there is another homothety, namely the uniform rescaling of the coordinates

$$
\left(z^{+}, z^{-}, z^{a}\right) \rightarrow \lambda\left(z^{+}, z^{-}, z^{a}\right) .
$$

(this is a combination of the first homothety with the boost). Since Brinkmann coordinates are Fermi coordinates [27] and thus a direct measure of geodesic distances, this is indeed a physical scale transformation. Thus in this case, one can complete the Seiberg-CSV procedure in a natural way by a rescaling of the coordinates and the metric exactly as in (2.26),

$$
\hat{z}^{\mu}=\epsilon z^{\mu} \quad d \hat{s}^{2}=\epsilon^{2} d s^{2} .
$$

In Rosen coordinates, this transformation of the coordinates reads

$$
\left(\hat{x}^{+}, \hat{x}^{-}, \hat{x}^{i}\right)=\left(\epsilon x^{+}, \epsilon x^{-}, \epsilon^{1-m_{i}} x^{i}\right) .
$$

Thus, to summarise, there appears to be a straightforward and very natural extension of the Seiberg-CSV procedure to scale-invariant plane waves, in which the flat space Lorentz transformations (null rotation, boost) are implemented by isometries of the metric, and the Seiberg scaling is realised by a uniform scaling of the Brinkmann coordinates

We are thus now in the position to define, for a power-law plane wave metric of the form

$$
d s^{2}=-2 d y^{+} d y^{-}+g_{11}\left(y^{+}\right)\left(d y^{1}\right)^{2}+\ldots=-2 d y^{+} d y^{-}+\left(y^{+}\right)^{2 m_{1}}\left(d y^{1}\right)^{2}+\ldots
$$

the DLCQ Hamiltonian, as before, via

$$
H_{N}^{D L C Q}(m, R):=\lim _{R_{s} \rightarrow 0} i \partial_{y^{+}} .
$$


Performing the null rotation isometry (2.33) with parameter $\alpha$, the boost isometry (2.36) with parameter $\gamma$, and the homothety (2.41) with parameter $\epsilon$ (momentarily treating these parameters as independent), one finds, as the generalisation of (2.20) (and with the final scaling already performed)

$$
\partial_{y^{+}}=\epsilon \gamma\left[\partial_{\hat{x}^{+}}-(\alpha / \gamma)(\epsilon \gamma)^{m_{1}} g^{11}\left(\hat{x}^{+}\right) \partial_{\hat{x}^{1}}+\left(\alpha^{2} / 2 \gamma^{2}\right)(\epsilon \gamma)^{2 m_{1}} g^{11}\left(\hat{x}^{+}\right) \partial_{\hat{x}^{-}}\right]
$$

We see that this has a finite limit as $\epsilon \rightarrow 0\left(R_{s} \rightarrow 0\right)$, provided that $\gamma \sim \alpha \sim \epsilon^{-1}$. We also see that, for non-contant $g_{11}$ there is no choice of constant parameters $\alpha, \gamma$ for which the rhs reduces directly to the background subtracted energy, namely $\hat{E}-\sqrt{g^{11}\left(\hat{x}^{+}\right)} \hat{p}_{1}$, arising from the appropriate generalisation of $(2.9)$,

$$
\hat{E}=\sqrt{g^{11}\left(\hat{x}^{+}\right)\left(\hat{p}_{1}\right)^{2}+\overrightarrow{\hat{p}}^{2}+m^{2}} \quad \Rightarrow \quad \hat{E}-\sqrt{g^{11}\left(\hat{x}^{+}\right)} \hat{p}_{1}=\frac{\overrightarrow{\hat{p}}^{2}+m^{2}}{2 \sqrt{g^{11}\left(\hat{x}^{+}\right)} \hat{p}_{1}}+\ldots
$$

However, as discussed at the end of section 2.2, it is still meaningful to use the above equation at the level of gauge-fixed energy fluctuations for any choice of $\alpha, \gamma, \epsilon$, subject to the condition $\gamma \sim \alpha \sim \epsilon^{-1}$, and we will henceforth make the simple choice (2.28)

$$
\alpha=\gamma=\epsilon^{-1}=\frac{R}{R_{s}},
$$

leading to $\delta E_{l c} \sim \delta \hat{E}$. With this choice of parameters, the combined action of the boost (2.36) and the rescaling (2.41) is the transformation

$$
\left(\tilde{x}^{+}, \tilde{x}^{-}, \tilde{x}^{i}\right)=\left(\hat{x}^{+}, \epsilon^{-2} \hat{x}^{-}, \epsilon^{-1} \hat{x}^{i}\right),
$$

which, as noted before, is actually a homothety (2.38) for any plane wave metric. In particular, the original lightcone time coordinate $y^{+}$is equal to the "Yang-Mills" lightcone gauge time variable $\hat{x}^{+}=\tau$. Moreover, combined with the rescaling (2.40) of the metric, this is precisely the scaling that defines the Penrose plane wave limit $[36,37,38]$. In the present case of plane waves, the Penrose limit leaves the metric invariant, and this is precisely as it should be since the DLCQ procedure should ideally not deform the metric (or other background fields).

Obviously, once one thinks of this combined transformation, one is naturally led to explore the relationship between DLCQ and the Penrose limit for more general backgrounds, e.g. as in [39] or $[40,41]$. However, it is not clear to us whether the result should then really be thought of as the DLCQ of a theory in the original background (this appears to be the point of view adopted e.g. in [40]) rather than as a DLCQ of a theory in some Penrose plane wave limit (depending on a choice of null geodesic) of the original background.

As a final remark, we should also point out that in some of the post-CSV literature dealing with the extension of the CSV model to curved backgrounds (typically plane waves, even though not always recognised as such, written in almost-Rosen coordinates like (A.2,A.3)), the boost and scale transformation of the Seiberg-Sen procedure were implemented by the naive flat space boost

$$
\left(y^{+}, y^{-}, y^{i}\right) \rightarrow\left(\epsilon y^{+}, \epsilon^{-1} y^{-}, y^{i}\right)
$$

and the naive flat space scaling

$$
\left(y^{+}, y^{-}, y^{i}\right) \rightarrow \epsilon^{-1}\left(y^{+}, y^{-}, y^{i}\right) .
$$


Now the former is not an isometry of a plane wave metric (not even when it is of the powerlaw, singular homogeneous, type), and the latter is neither a homothety nor a physical scale transformation of the metric (Rosen coordinates are not a measure of proper physical distance in space-time). Nevertheless, the combined action of these two transformations happens to be identical to the combined action (2.47) resulting from the boost isometry (2.36) and the physical scale transformation (2.41), and thus in the present case one can get away with this. However, it should be clear from what we have said that conceptually at least this appears to be an incorrect implementation of DLCQ, or at least one that requires further justification. Our more systematic treatment of the DLCQ will also lead to a quite different, and significantly simpler, analysis of decoupling conditions and related issues.

\subsection{9/11 Flip, DBI Expansion, and Decoupling}

We have now prepared the ground for the DLCQ of IIA string theory in singular homogeneous plane wave - null dilaton backgrounds such as those determined in appendix B, (B.9). We assume that we have already performed the null rotation $y^{\mu} \rightarrow \tilde{x}^{\mu}$ aligning the almost null circle with the spatial direction $\tilde{x}^{1}$, such that $\tilde{x}^{1} \sim \tilde{x}^{1}+2 \pi R_{s}$, and we focus on a sector with $N$ units of momentum $\tilde{p}_{1}=N / R_{s}$.

Via the procedure outlined at the end of section 2.1 (boosting and scaling, lifting to M-theory along $x^{11}$ with scaled radius $\hat{R}_{11}=\epsilon \ell_{s} g_{s}, \ell_{s}$ and $g_{s}$ denoting the original IIA string length and string coupling respectively, then reducing along the scaled circle with radius $\hat{R}_{1}=\hat{R}_{s}=\epsilon^{2} R$, and performing a T-duality along $x^{11}$ ), one arrives at a definition of the DLCQ of IIA string theory in terms of a (decoupling) limit of IIB string theory in a sector with $N$ D1-branes.

Alternatively, one can use the 9/11 flip [4,5] (more appropriately called a 1/11 flip in the present context) to arrive at the same theory (in entirely 10-dimensional terms) by performing first the boost and scaling, then a T-duality along $\hat{R}_{1}$ to IIB with $N$ fundamental string winding modes, and then an S-duality to IIB with $N$ D1-branes. In this way one arrives at the same description of the DLCQ of IIA string theory. This equivalence is of course well known in principle, and we emphasise it here only because in [8] the scaling was only performed after the TS-duality, and even then only somewhat implicitly (in the definition of the Yang-Mills time variable). In general one has to perform the scaling rightaway, in conjunction with the boost, to obtain equivalence with the first prescription (which is rooted firmly in the Seiberg-Sen derivation of the BFSS matrix theory).

Following this procedure, one finds that the parameters of the final IIB string theory are related to the $\ell_{s}, g_{s}$ and $R_{11}=\ell_{s} g_{s}$ of the original IIA theory by (we will denote IIB quantities by a prime)

$$
\left(\ell_{s}^{\prime}\right)^{2}=\epsilon\left(\ell_{s}\right)^{2} \frac{R_{11}}{R} \quad g_{s}^{\prime}=\epsilon \frac{R}{R_{11}},
$$

and that the scaled ST-dual metric-dilaton background is given by

$$
\begin{aligned}
\left(d s^{\prime}\right)^{2} & =\sqrt{g_{11}} \mathrm{e}^{-\phi}\left(-2 d \hat{x}^{+} d \hat{x}^{-}+\frac{\ell_{s}^{4}}{R^{2} g_{11}}\left(d \hat{x}^{1}\right)^{2}+\ldots\right) \equiv \mathrm{e}^{\phi^{\prime}} d \tilde{s}^{2} \\
\phi^{\prime} & =-\phi+\frac{1}{2} \log g_{11}
\end{aligned}
$$


where $\phi$ and $g_{11}$ are functions of $\hat{x}^{+}=\tilde{x}^{+}=y^{+}$(the original lightcone coordinate) and $d \tilde{s}^{2}$ is the T-dual of the original scaled IIA metric. Note that this is again a plane wave metric, written in the almost-Rosen coordinates (A.2). It is neither necessary nor convenient to introduce a true Rosen +-coordinate at this stage since the prefactor of the above metric will in any case drop out of the DBI action to be discussed below. We have normalised $\hat{x}^{1}$ to have unit radius, $\hat{x}^{1} \sim \hat{x}^{1}+2 \pi$. Note that it is due to the fact that we have implemented the boost by isometries of the metric, and that we have already performed the scaling, that neither the metric nor the dilaton has any (undesirable) explicit $\epsilon$-dependence.

The next step is to look at the Abelian $(N=1)$ D1-brane DBI action in the background $(2.51)$,

$$
S=-\frac{1}{2 \pi g_{s}^{\prime} \ell_{s}^{\prime 2}} \int d \tau d \sigma \mathrm{e}^{-\phi^{\prime}} \sqrt{-\operatorname{det}\left(\partial_{\alpha} \hat{x}^{\mu} \partial_{\beta} \hat{x}^{\nu} g_{\mu \nu}^{\prime}+2 \pi \ell_{s}^{\prime 2} F_{\alpha \beta}\right)}
$$

and to expand the fields to quadratic order around a suitable classical solution of this action. First of all we observe that

$$
g_{s}^{\prime} \ell_{s}^{\prime 2}=\left(\epsilon \ell_{s}\right)^{2}
$$

the scaled original string length squared, and that, correspondingly, the dilaton in the DBI action reconverts the ST-dual metric to the T-dual metric $d \tilde{s}^{2}$ implicitly defined in (2.51). We now seek a solution $\hat{x}_{c}^{\mu}$ of the DBI equations of motion that describes the groundstate of a simply wrapped D1-string around the $\hat{x}^{1}$-direction. Thus we make the lightcone gauge ansatz

$$
\hat{x}_{c}^{+}=a \tau \quad, \quad \hat{x}_{c}^{1}=b \sigma \quad, \quad \hat{x}_{c}^{m}=0 \quad, \quad\left(A_{\alpha}\right)_{c}=0 .
$$

The solution for $\hat{x}_{c}^{-}$can then be found by integrating the lightcone gauge constraint equations $\partial_{\sigma} \hat{x}_{c}^{-}=0$ and $\partial_{\tau} \hat{x}_{c}^{-}=b^{2} \tilde{g}_{11} / 2 a$. Compatibility of the classical solution with the periodicity of $\hat{x}^{1}$ and the choice $\sigma \sim \sigma+2 \pi \ell_{s}$ fixes $b=1 / \ell_{s}$, and we may as well also choose $a=1$. Choosing now the gauge $\hat{x}^{+}=\hat{x}_{c}^{+}, \hat{x}^{1}=\hat{x}_{c}^{1}$, the fluctuations are the gauge fields and the fields $\left(X^{1}, X^{m}\right)$ defined (with a convenient normalisation) by

$$
\hat{x}^{-}(\tau, \sigma)=\hat{x}_{c}^{-}(\tau)+\epsilon \frac{\ell_{s}}{R} X^{1}(\tau, \sigma) \quad, \quad \hat{x}^{m}(\tau, \sigma)=\epsilon X^{m}(\tau, \sigma) .
$$

The fluctuation expansion then becomes an $\epsilon$-expansion (compatible with the $\epsilon^{-2}$-prefactor arising from (2.53)), and to quadratic order in the fluctuations one finds, after an unenlightning but straightforward calculation, dropping the field-independent classical action and a total derivative term, the action

$$
S=\frac{1}{2 \pi \ell_{s}^{2}} \int d \tau d \sigma\left[\frac{1}{2} g_{i j}(\tau)\left(\partial_{\tau} X^{i} \partial_{\tau} X^{j}-\partial_{\sigma} X^{i} \partial_{\sigma} X^{j}\right)+2 \pi^{2} \ell_{s}^{4} g_{s}^{2} \mathrm{e}^{2 \phi(\tau)} F_{\tau \sigma}^{2}\right] .
$$

The final result is extremely simple. All that enters, after this sequence of manipulations, are the transverse metric components and the dilaton of the original IIA configuration, not its Tdualised or S-dualised cousins. In particular, the coupling constant of the gauge theory is set by the original dilaton,

$$
g_{Y M} \sim \frac{1}{g_{s} \ell_{s}} \mathrm{e}^{-\phi} .
$$

Moreover, in complete generality the field $X^{1}$, which began life as a fluctuation of $\hat{x}^{-}$, in the end plays the role of $x^{1}$ (which itself had been gauge fixed). The $\epsilon$-scaling of the fluctuations 
in (2.55) is also natural from this point of view, since it undoes the Penrose scaling (2.47) of the transverse coordinates $\tilde{x}^{i}$, so that the fluctuations are directly related to the coordinates of the original metric (just as the choice $a=1$ identifies the worldsheet time coordinate with the original lightcone time coordinate).

It remains to discuss the validity of the truncation of IIB string theory in the sector with 1 D-brane $(N=1)$ to the above action. To that end, recall first that we had already established in section 2.3 that at the level of gauge fixed fluctuations (the situation we are dealing with here), the energies $\delta \hat{E}$ of the above fluctuation action are finite and equal to the string theory lightcone energy fluctuations. Thus in order to establish the decoupling of massive open strings and bulk closed string modes, we need to compare their energies with the YM energies in the limit $\epsilon \rightarrow 0$.

In principle, following [8], this could be accomplished by defining a suitable effective string or Planck length (incorporating the effect of the non-trivial IIB dilaton). However, this is unnecessary since the metric and dilaton of the final IIB theory are in any case independent of $\epsilon$ and thus have no bearing on the issue of decoupling in the DLCQ limit $\epsilon \rightarrow 0 .^{3}$ The $\epsilon$-dependence resides only in the IIB string length and string coupling (2.50), both of which go to zero as $\epsilon \rightarrow 0$. It follows that

$$
\lim _{\epsilon \rightarrow 0}(\delta \hat{E}) \ell_{s}^{\prime}=\lim _{\epsilon \rightarrow 0}(\delta \hat{E})\left(g_{s}^{\prime}\right)^{1 / 4} \ell_{s}^{\prime}=0
$$

which establishes the decoupling of massive open and closed string modes.

\section{Some Basic Properties of the Matrix String Action for SHPWs}

\subsection{The Plane Wave Matrix String Action in Rosen Coordinates}

It is now reasonable to assume that, in the absence of background fields other than the metric and the dilaton, the non-Abelian version of the above action, i.e. the decoupled action arising from the sector of IIB string theory with $N$ would D1-strings, is given by the obvious nonabelianisation of the above action. ${ }^{4}$ Thus, more or less following the usual matrix string theory conventions (numerical factors can be changed by various scalings of the fields and coordinates) and with the insight that the Yang-Mills coupling constant in this model is set by the dilaton via (2.57), the bosonic part of the matrix string action is (the $X^{i}=X^{i}(\tau, \sigma)$ now denote hermitian matrix valued fields)

$$
\begin{aligned}
S=\frac{1}{2 \pi \ell_{s}^{2}} \int d \tau d \sigma \operatorname{Tr}\left\{\frac{1}{2} g_{i j}(\tau)\left(D_{\tau} X^{i} D_{\tau} X^{j}-D_{\sigma} X^{i} D_{\sigma} X^{j}\right)+2 \pi^{2} \ell_{s}^{4} g_{s}^{2} \mathrm{e}^{2 \phi(\tau)} F_{\tau \sigma}^{2}\right. \\
\left.+\frac{1}{16 \pi^{2} \ell_{s}^{4} g_{s}^{2}} \mathrm{e}^{-2 \phi(\tau)} g_{i k}(\tau) g_{j l}(\tau)\left[X^{i}, X^{j}\right]\left[X^{k}, X^{l}\right]\right\} .
\end{aligned}
$$

\footnotetext{
${ }^{3}$ In this respect, our analysis differs from that of [8] and, in particular, subsequent articles that claimed to find a much more complicated $\epsilon$-dependence arising from the metric and dilaton.

${ }^{4}$ The presence of other background fields would complicate matters due to the appearance of Myers terms etc.
} 
In terms of the flat worldsheet metric $\eta_{\alpha \beta}$ this can be written as

$$
\begin{aligned}
S=\frac{1}{2 \pi \ell_{s}^{2}} \int d^{2} \sigma \operatorname{Tr}\left\{-\frac{1}{2} \eta^{\alpha \beta} g_{i j}(\tau)\right. & D_{\alpha} X^{i} D_{\beta} X^{j}-\frac{1}{4} 4 \pi^{2} \ell_{s}^{4} g_{s}^{2} \mathrm{e}^{2 \phi(\tau)} \eta^{\alpha \gamma} \eta^{\beta \delta} F_{\alpha \beta} F_{\gamma \delta} \\
& \left.+\frac{1}{4} \frac{1}{4 \pi^{2} \ell_{s}^{4} g_{s}^{2}} \mathrm{e}^{-2 \phi(\tau)} g_{i k}(\tau) g_{j l}(\tau)\left[X^{i}, X^{j}\right]\left[X^{k}, X^{l}\right]\right\} .
\end{aligned}
$$

This is the matrix string action for a plane wave in Rosen coordinates, a convenient coordinate system to start off with since the isometry directions required for the reductions, T-duality etc., were manifest. However, for many purposes Brinkmann coordinates are more convenient, and we will discuss the Brinkmann version of this action below, since it also has several advantages over the Rosen coordinate action.

At this point it is appropriate to say a few words about the fermionic part of these actions. Apart from the standard kinetic term and Yukawa couplings one may ask if there are any other additional terms arising from the non-trivial target-space metric. Such additional terms arise from the spin connection contribution to the covariant derivative and have been discussed for D-brane actions for instance in [42]. They are typically of the form

$$
\bar{\Psi} \Gamma^{\alpha} \omega_{\alpha}^{M N} \Gamma_{M N} \Psi
$$

where $\alpha$ are world-volume indices, the $\Psi$ are Majorana spinors and the $\Gamma$ real $32 \times 32$ gamma matrices (perhaps with a projection on the spinors, depending on the target space string theory).

In order to calculate the spin-connection for a plane wave metric in Rosen coordinates, we introduce the orthonormal frame $E^{ \pm}=d y^{ \pm}, E^{a}=E_{i}^{a} d y^{i}$ with $E_{i}^{a}$ a vielbein for $g_{i j}\left(y^{+}\right)$. The calculation becomes particularly simple when one chooses the special (parallel) frame that also happens to enter in the transformation from Rosen to Brinkmann coordinates (A.8) and which satisfies the symmetry condition (A.9). Then one finds that the only non-vanishing components of the spin connection are

$$
\omega^{-a}=\dot{E}_{I}^{a} d x^{i} .
$$

In particular, these have no components in the worldsheet directions that could contribute to (3.3). Although we have used the special symmetric frame to do this calculation, the term that we are considering (plus the suppressed fermion kinetic term) is covariant under frame rotations, and the result is thus independent of the choice of frame.

In principle, there are also dilatonic contributions to the D-brane action [42] which, in the absence of RR fields, take the simple form

$$
\bar{\Psi} \Gamma^{\alpha} \partial_{\alpha} \phi \Psi
$$

For Majorana fermions, with $\bar{\Psi}=\Psi^{T} \Gamma^{0}$ in a Majorana basis, say, this term is also zero since $\Gamma^{0} \Gamma^{\alpha}$ is symmetric. ${ }^{5}$ Thus in our case there are no additional fermionic contributions to the D-string action arising form either the space-time metric or the dilaton.

Since the bosonic part of the matrix string action (3.2) can be regarded as the dimensional reduction of the 10d Yang-Mills action in the Rosen coordinate plane wave background to $1+1$

\footnotetext{
${ }^{5}$ The presence of RR fields would have contributed additional projectors to (3.5), which could then have given rise to potentially non-zero contributions of the dilaton gradient to the D-brane action.
} 
dimensions (along the transverse translational isometries of the plane wave), one might perhaps have expected the presence of other terms of the same form as (3.3) but with a contraction over transverse space-time indices $i$ rather than the world-volume indices $\alpha$ in the fermionic part of the matrix string action. While the analysis of [42] suggests that such terms should not appear in the action, they in any case also turn out to be identically zero for plane waves. Namely, using (3.4) and once again the symmetry condition (A.9), one finds that the only possible further contribution to the action is

$$
\bar{\Psi} E_{A}^{I} \dot{E}_{I}^{A} \Gamma^{+} \Psi
$$

This term, however, is zero for anti-commuting Majorana fermions, for the same reasons as above.

We close this section with one remark regarding the generality of the above action. Strictly speaking, we have derived this action only for singular homogeneous plane waves since in that case we could implement almost literally the accepted flat space Seiberg-Sen DLCQ prescription (in its CSV variant). However, as it stands, this action makes sense for any plane wave metric - dilaton system. If one can argue that, in order to correctly implement the DLCQ in curved backgrounds, one only needs to consider the combined boost-scaling Penrose transformation (2.47), as e.g. the arguments in [39] suggest, then the entire derivation of the matrix string action, including the decoupling arguments, still goes through, and one would then have estabished the validity of the above matrix string action for any plane wave.

\subsection{Rosen vs Brinkmann Form of the Matrix String Action}

Here we discuss the Brinkmann coordinate (BC) counterpart of the Rosen coordinate (RC) matrix Yang-Mills action (3.2), which we now write in slightly simplified form as

$$
\begin{aligned}
S_{R C}=\int d^{2} \sigma \operatorname{Tr}\left(-\frac{1}{4} g_{Y M}^{-2} \eta^{\alpha \gamma} \eta^{\beta \delta} F_{\alpha \beta} F_{\gamma \delta}-\frac{1}{2} \eta^{\alpha \beta} g_{i j}(\tau) D_{\alpha} X^{i} D_{\beta} X^{j}\right. & \\
& \left.+\frac{1}{4} g_{Y M}^{2} g_{i k}(\tau) g_{j l}(\tau)\left[X^{i}, X^{j}\right]\left[X^{k}, X^{l}\right]\right)
\end{aligned}
$$

Now in Brinkmann coordinates a plane wave takes the form (A.6),

$$
-2 d x^{+} d x^{-}+g_{i j}\left(x^{+}\right) d x^{i} d x^{j}=-2 d z^{+} d z^{-}+A_{a b}\left(z^{+}\right) z^{a} z^{b}\left(d z^{+}\right)^{2}+\delta_{a b} d z^{a} d z^{b}
$$

and since typically (e.g. in the lightcone gauge point particle or string actions) $A_{a b}$ turns into a mass term for the fields, as a potential Brinkmann counterpart of this action, we consider the action

$$
\begin{aligned}
S_{B C}=\int d^{2} \sigma \operatorname{Tr}\left(-\frac{1}{4} g_{Y M}^{-2} \eta^{\alpha \gamma} \eta^{\beta \delta} F_{\alpha \beta} F_{\gamma \delta}-\frac{1}{2} \eta^{\alpha \beta} \delta_{a b} D_{\alpha} Z^{a} D_{\beta} Z^{b}\right. & \\
& \left.+\frac{1}{4} g_{Y M}^{2} \delta_{a c} \delta_{b d}\left[Z^{a}, Z^{b}\right]\left[Z^{c}, Z^{d}\right]+\frac{1}{2} A_{a b}(\tau) Z^{a} Z^{b}\right)
\end{aligned}
$$

On the face of it, these two classes of actions appear to be rather different, with (3.7) having non-standard time-depenent kinetic terms and quartic couplings for the scalar fields, described by the $g_{i j}(t)$, while (3.9) has standard kinetic and quartic terms but time-dependent mass terms for the scalars (with $A_{a b}(t)$ minus the mass-squared matrix). Nevertheless, we claim that 
these two types of Yang-Mills actions are simply related by a certain linear field redefinition $X^{i}=E_{a}^{i}(\tau) Z^{a}$ of the scalar fields,

$$
S_{R C}\left[A_{\alpha}, X^{i}=E_{a}^{i} Z^{a}\right]=S_{B C}\left[A_{\alpha}, Z^{a}\right] .
$$

To see this, recall first of all the coordinate transformation (A.8) between Rosen and Brinkmann coordinates, in particular the part $x^{i}=E_{a}^{i} z^{a}$. We are thus led to consider the linear field transformation

$$
X^{i}(\tau, \sigma)=E_{a}^{i}(\tau) Z^{a}(\tau, \sigma)
$$

of the scalar fields (matrix-valued coordinates) $X^{i}$ and $Z^{a}$, where $E_{a}^{i}$ is a vielbein for the time-dependent metric $g_{i j}(\tau)$ on the scalar field space satisfying the symmetry condition (A.9). Substituting (3.11) into the RC Lagrangian $L_{R C}$, one can now verify that one indeed obtains the BC Lagrangian $L_{B C}$ up to a total time-derivative (related to the shift of $x^{-}$in (A.8)). We explain this calculation in somewhat more detail in [29], where we also show that this kind of argument extends to the plane wave counterparts of the recently proposed multiple M2-brane actions [30, 31, 32] based on 3-algebras rather than Lie algebras. Here we just want to point out that a crucial role in this calculation is played by the symmetry condition (A.9) (and, of course, by the gauge invariance of the action). Not only is this condition responsible for several cancellations that are akin to those that already occur in the transformation of a plane wave metric from Rosen to Brinkmann coordinates. It cooperatively also serves to eliminate some terms of genuinely non-Abelian origin, such as

$$
g_{i j}(t) E_{a}^{i} \dot{E}_{b}^{j} \operatorname{Tr}\left[A_{t}, Z^{a}\right] Z^{b}=g_{i j}(t) E_{a}^{i} \dot{E}_{b}^{j} \operatorname{Tr} A_{t}\left[Z^{a}, Z^{b}\right]=0
$$

arising from the scalar kinetic terms. The above equivalence (3.10) is also valid for a timedependent coupling constant $g_{Y M}(\tau)$, since the total time-derivative arises only from the dilatonindependent scalar kinetic term. It also extends to the fermionic terms in the action in a rather trivial way, since the (Yukawa) coupling between the fermions and the scalars $X^{i}$ is purely algebraic.

The main advantage of the Brinkmann form (3.9) of the action is that the scalar fields have standard kinetic terms. This implies that it is legitimate and meaningful to look at the potential terms to deduce some properties of the classical and quantum theories. In particular, whether one is in an Abelian or non-Abelian phase of the theory can be reliably read off from the behaviour of the dilaton. For example, when the dilaton blows up at the singularity (a strong coupling singularity in the sense of the analysis in appendix B.3), the Yang-Mills coupling constant (2.57) is small and one is in a genuinely non-Abelian phase of the theory, just as in the CSV model, where the matrix coordinates are non-commutative. By contrast, conclusions based solely on the analysis of the quartic potential term in the RC action (3.7), or the attempt to read off something like an effective string tension from the $\mathrm{RC}$ kinetic term, are bound to be misleading at best.

Furthermore, the mass terms in the $\mathrm{BC}$ action contain direct invariant geometric information about the space-time, since they arise from the components of the Riemann tensor in Brinkmann coordinates. In particular, we will see below that they faithfully encode the information whether 
one is dealing with a strong or weak coupling singularity (in the sense of the analysis in appendix B.3), something that is not at all manifest in the RC action which also exhibits spurious coordinate singularities.

\subsection{Absorbing the Coupling Constant into the Worldsheet Metric}

It is obvious, and a basic property of 2-dimensional gauge theories, that the dilaton / Yang-Mills coupling constant can, in either the Rosen or the Brinkmann form of the Yang-Mills action, in principle always be absorbed into a non-trivial worldsheet metric via

$$
h_{\alpha \beta}=\mathrm{e}^{-2 \phi} \eta_{\alpha \beta}
$$

since one then has

$$
\sqrt{h}=\mathrm{e}^{-2 \phi} \quad \sqrt{h} h^{\alpha \beta}=\eta^{\alpha \beta} \quad \sqrt{h} h^{\alpha \gamma} h^{\beta \delta}=\mathrm{e}^{2 \phi} \eta^{\alpha \gamma} \eta^{\beta \delta},
$$

which are precisely the pre-factors of the quartic, scalar kinetic and $F^{2}$ terms respectively. Once one has absorbed the dilaton into the worldsheet metric (in view of the considerations below it is not clear if one really wants to do this in general), the only time-dependence remaining in the Brinkmann coordinate matrix string action is in the mass terms $A_{a b}(\tau)$. The Rosen coordinate matrix string theory action, on the other hand, still has explicit time-dependence arising from the metric coefficients $g_{i j}(\tau)$. Such a remaining time-dependence can never be absorbed by a further (conformal) redefinition of the worldsheet metric since the combination $\sqrt{h} h^{\alpha \beta}$ is conformally invariant so that the time-dependence in the kinetic term $g_{i j} D X^{i} D X^{j}$ can not be eliminated in this way.

In the CSV model (flat metric with a linear dilaton), (3.13) resulted in a useful alternative description of the theory, heavily made use of e.g. in [9]. Instead of a Yang-Mills theory with a time-dependent coupling constant on a cylindrical worldsheet with the trivial metric one then has a Yang-Mills theory with a time-independent coupling constant on a cylindrical worldsheet with a non-trivial time-dependent metric. With $\phi=-Q \tau$, the line-element is

$$
d s^{2}=\mathrm{e}^{2 Q \tau}\left(-d \tau^{2}+d \sigma^{2}\right) .
$$

While this metric is locally flat, the periodicity of $\sigma$ results in the worldsheet being the Milne orbifold [8].

For the backgrounds with $b \neq-1$, on the other hand, we have exp $2 \phi(\tau)=\tau^{3 b /(b+1)}$. Thus the rescaled worldsheet metric has the form

$$
d s^{2}=\tau^{2 \gamma}\left(-d \tau^{2}+d \sigma^{2}\right)
$$

where $2 \gamma=-3 b /(b+1)$. The Einstein-dilaton equations imply that $\gamma \geq-1$ (B.14). Now this metric is singular even prior to the periodic identification of $\sigma$ unless either, trivially $\gamma=0$ (i.e. $b=0$, a constant dilaton), or $\gamma=-1$. Indeed, when $\gamma \neq-1$, the metric has a curvature singularity at $\tau=0$, as can be seen by calculating e.g. the Ricci scalar $R=-2 \gamma \tau^{-(2 \gamma+2)}$. For $\gamma=-1$, on the other hand, $R$ is constant and with $T=\log \tau$ one has

$$
d s^{2}=\tau^{-2}\left(-d \tau^{2}+d \sigma^{2}\right)=-d T^{2}+\mathrm{e}^{-2 T} d \sigma^{2} .
$$


This is just the $(1+1)$-dimensional de Sitter $(\mathrm{dS})$ metric, written in coordinates that cover half of the entire dS space-time. $\gamma=-1$ corresponds to $3 b /(b+1)=2$, i.e. $b=2$. This is the dual background (under the $b \rightarrow 1-b$ isometry) of the CSV solution (see the remark after (B.18)), and thus the $\mathrm{dS}$ worldsheet arises in the dual reduction of the CSV M-theory background (B.3).

Periodicity of $\sigma$ means that we are considering here dS space-time with toroidal (rather: circular) spatial sections. While on the face of it this appears to be an innocuous modification of the dS metric, this space-time is, in spite of apparently being non-singular, actually known to be timelike geodesically incomplete $[43,44]$ (i.e. it contains inextendible timelike geodesics of finite length). Note that this worldsheet geodesic incompleteness appears at $T \rightarrow-\infty$, i.e. at the location of the space-time singularity. Nevertheless, this worldsheet structure may be more tractable than the (genuinely singular) worldsheets that arise for $\gamma \neq-1$. In particular, here the point of incompleteness is "inaccessible" in the sense that an observer who wants to reach it in finite proper time needs to wrap around the circle an infinite number of times.

\subsection{Tachyons and Strong String Coupling Singularities}

Let us take a closer look at the scalar sector of the BC action (3.9). The information about the metric resides solely in the mass matrix

$$
\mu_{a b}^{2}(\tau)=-A_{a b}(\tau)
$$

For the singular homogeneous plane wave backgrounds of Appendix B one has (B.9)

$$
\mu_{a b}^{2}(\tau)=\mu_{a}^{2}(\tau) \delta_{a b}=-m_{a}\left(m_{a}-1\right) \tau^{-2} \delta_{a b} .
$$

Now by the Einstein-dilaton equation (B.13),

$$
\sum_{a} m_{a}\left(m_{a}-1\right)=-\frac{3 b}{b+1}
$$

the parameters $m_{a}$ are related to the parameter $b$ determining the dilaton $\phi$. The sign of $b$ in turn determines (appendix B.3) whether $\exp \phi$ blows up at the singularity (strong coupling singularity, $b<0$ ) or goes to zero there (weak coupling singularity, $b>0$ ). In particular, if all the mass-squares $\mu_{a}^{2}$ of the scalars are positive, necessarily $b$ is positive, and one is dealing with a weak coupling singularity. Conversely, therefore, whenever one is dealing with a strong coupling singularity, at least one of the scalars is tachyonic (and the sum over all the $\mu_{a}^{2}$ is negative). Thus this is the way a strong coupling singularity manifests itself in the BC matrix string action.

The derivation of the model, in particular the decoupling analysis of section 2.4, suggests that the matrix string action gives a valid description of the string theory at least for all $\tau>0$ and even when $\tau \rightarrow 0$. Thus the presence of these tachyonic mass terms, which can in principle occur both for strong and for weak coupling singularities, should not all by itself be indicative of a pathology of the model. ${ }^{6}$ In particular, in the non-Abelian phase of the theory the tachyonic mass terms

\footnotetext{
${ }^{6}$ In this context it may be worth pointing out that in a related setting, namely the modelling of cosmological singularities via AdS/CFT, it was also found to be necessary to introduce a potential that is unbounded from below [45]. More recently, tachyonic mass terms of the above $\tau^{-2}$-type have also been shown to arise naturally in the cosmological AdS/CFT context [46].
} 
can potentially be stabilised by the quartic potential, perhaps indicating the existence of some new and interesting non-perturbative physics. One might e.g. like to see if there is a qualitatively different behaviour for strong $\left(\sum_{a} \mu_{a}^{2}<0\right)$ vs weak $\left(\sum_{a} \mu_{a}^{2}>0\right)$ coupling singularities. It may also be of interest to study the implications of the classical scale invariance of these models, manifested e.g. in the characteristic $\tau^{-2}$-dependence of the mass terms, in the quantum theory.

\section{ACKNOWLEDGEMENTS}

We are very grateful to Denis Frank, Giuseppe Milanesi and Sebastian Weiss for numerous helpful discussions during the long gestation-period of this project, and for their collaboration on related matters. This work has been supported by the Swiss National Science Foundation and by the EU under contract MRTN-CT-2004-005104.

\section{A Plane Wave Geometry: Synopsis}

There are two standard coordinate systems for plane wave metrics, each with its own advantages. In Rosen coordinates, the metric takes the form

$$
d s^{2}=g_{\mu \nu} d y^{\mu} d y^{\nu}=-2 d y^{+} d y^{-}+g_{i j}\left(y^{+}\right) d y^{i} d y^{j} .
$$

In Rosen coordinates it is manifest that any metric conformal to a plane wave metric, with the conformal factor depending only on $y^{+}$,

$$
d s^{2}=f\left(y^{+}\right)\left(-2 d y^{+} d y^{-}+g_{i j}\left(y^{+}\right) d y^{i} d y^{j}\right),
$$

or, equivalently, a metric of the type

$$
d s^{2}=-2 f\left(y^{+}\right) d y^{+} d y^{-}+g_{i j}\left(y^{+}\right) d y^{i} d y^{j},
$$

is again a plane wave metric, as can be sen by defining the new Rosen coordinate (affine parameter) $\tilde{y}^{+}$by $d \tilde{y}^{+}=f\left(y^{+}\right) d y^{+}$.

The metric (A.1) has the manifest commuting translational Killing vectors $Z=\partial_{y^{-}}$and $Q_{(i)}=$ $\partial_{y^{i}}$. In addition, any plane wave metric has the "hidden" dual commuting translational Killing vectors

$$
P^{(i)}=y^{i} \partial_{y^{-}}+h^{i k} \partial_{y^{k}}
$$

where

$$
h^{i k}\left(y^{+}\right)=\int^{y^{+}} d u g^{i k}(u)
$$

which extend the Abelian isometry algebra generated by $Q_{(i)}$ and $Z$ to the Heisenberg algebra $\left[Q_{(i)}, P^{(k)}\right]=\delta_{i}^{k} Z$ with central element $Z$.

Rosen coordinates are not the coordinate system in which plane waves are usually and most conveniently discussed, among other reasons because typically in Rosen coordinates the metric exhibits spurious coordinate singularities. The plane wave metric in Brinkmann coordinates is

$$
d s^{2}=g_{\mu \nu} d z^{\mu} d z^{\nu}=-2 d z^{+} d z^{-}+A_{a b}\left(z^{+}\right) z^{a} z^{b}\left(d z^{+}\right)^{2}+\delta_{a b} d z^{a} d z^{b} .
$$


Brinkmann coordinates are Fermi coordinates adapted to the null geodesic $\left(z^{+}=\tau, z^{-}=0, z^{a}=\right.$ 0) [27]. In particular, Brinkmann coordinates are, like Riemann coordinates, a direct measure of the invariantly defined geodesic distance in space-time.

Moreover, and related to this, in Brinkmann coordinates, the curvature of the plane wave is related purely algebraically to the mass/frequency term $A_{a b}(u)$ of the metric, which trivialises the task of calculating the curvature of a plane wave. Specifically, the only non-vanishing components of the Riemann tensor and Ricci tensor are

$$
R_{+a+b}\left(z^{+}\right)=-A_{a b}\left(z^{+}\right) \quad R_{++}\left(z^{+}\right)=-\delta^{a b} A_{a b}\left(z^{+}\right)
$$

and the Ricci scalar is zero. Thus the metric is flat iff $A_{a b}=0$ and the vacuum Einstein equations reduce to the simple algebraic condition on $A_{a b}$ (regardless of its $z^{+}$-dependence) that it be traceless. The number of degrees of freedom of this traceless matrix $A_{a b}\left(z^{+}\right)$are those of a transverse traceless symmetric tensor (a.k.a. a graviton).

The two classes of metrics described by (A.1) and (A.6) are equivalent: every metric of the form (A.1) can be brought to the form (A.6), and conversely every metric of the type (A.6) can be written, in more than one way, as in (A.1). The coordinate transformation relating (A.1) and (A.6) is

$$
\left(y^{+}, y^{-}, y^{i}\right)=\left(z^{+}, z^{-}-\frac{1}{2} \dot{E}_{a i} E_{b}^{i} z^{a} z^{b}, E_{a}^{i} z^{a}\right)
$$

where $E_{a}^{i}=E_{a}^{i}\left(y^{+}\right)$is a vielbein for $g_{i j}\left(y^{+}\right), E_{a}^{i} E_{b}^{j} g_{i j}=\delta_{a b}$, subject to the symmetry condition

$$
\dot{E}_{a i} E_{b}^{i}=\dot{E}_{b i} E_{a}^{i}
$$

(which can be interpreted $[23,24]$ as the condition that the frame $E_{a}^{i}$ is parallel transported along the congruence of null geodesics defined by the Rosen coordinates). The relation between $g_{i j}\left(y^{+}\right)$and $A_{a b}\left(z^{+}\right)$can be succinctly written as [22] (recall that $z^{+}=y^{+}$)

$$
A_{a b}\left(z^{+}\right)=\ddot{E}_{a i}\left(z^{+}\right) E_{b}^{i}\left(z^{+}\right)
$$

The above relations simplify considerably for diagonal metrics, $g_{i j}\left(y^{+}\right)=g_{i}\left(y^{+}\right)^{2} \delta_{i j}$, for which one simply has

$$
A_{a b}\left(z^{+}\right)=\frac{\ddot{g}_{a}\left(z^{+}\right)}{g_{a}\left(z^{+}\right)} \delta_{a b}
$$

In particular, for Rosen coordinate metrics of power-law type,

$$
d s^{2}=-2 d y^{+} d y^{-}+\sum_{i}\left(y^{+}\right)^{2 m_{i}}\left(d y^{i}\right)^{2},
$$

the metric in Brinkmann coordinates is

$$
d s^{2}=-2 d z^{+} d z^{-}+\sum_{a} \frac{m_{a}\left(m_{a}-1\right)}{\left(z^{+}\right)^{2}}\left(z^{a}\right)^{2}\left(d z^{+}\right)^{2}+\sum_{a}\left(d z^{a}\right)^{2}
$$

and its Ricci tensor is

$$
R_{++}\left(z^{+}\right)=\sum_{a} m_{a}\left(m_{a}-1\right)\left(z^{+}\right)^{-2}
$$

Since $m_{a}\left(m_{a}-1\right)$ is invariant under $m_{a} \rightarrow 1-m_{a}$, (A.13) shows that Rosen coordinate metrics with $m_{i}$ and $m_{i} \rightarrow 1-m_{i}$ are isometric. In particular, any metric with all $m_{i}=0,1$ is flat. 
It is evident e.g. from (A.14) that these plane waves are singular at $z^{+}=0$ and, since $z^{+}$can play the role of an affine geodesic parameter, that this singularity is at finite affine distance, so that these metrics are geodesically incomplete. Moreover, these power-law metrics and their Brinkmann coordinate counterparts have the special property that they are scale-invariant, i.e. invariant under scalings of the coordinate (affine parameter) $y^{+}$or $z^{+}$. This is evident for (A.13), which is invariant under

$$
\left(z^{+}, z^{-}\right) \rightarrow\left(\lambda z^{+}, \lambda^{-1} z^{-}\right)
$$

Thus these metrics have the additional Killing vector $X=z^{+} \partial_{z^{+}}-z^{-} \partial_{z^{-}}$. The corresponding isometry in Rosen coordinates (A.12) is

$$
\left(y^{+}, y^{-}, y^{i}\right) \rightarrow\left(\lambda y^{+}, \lambda^{-1} y^{-}, \lambda^{-m_{i}} y^{i}\right) .
$$

See [21] and [22] for a systematic discussion and other properties of these singular homogeneous plane waves.

\section{B A Class of Plane Wave - Null Dilaton Big Bang Backgrounds}

\section{B.1 M $\rightarrow$ IIA Reduction of Singular Homogeneous Plane Wave Backgrounds}

Using the standard relation

$$
d s_{11}^{2}=\mathrm{e}^{-2 \phi / 3} d s_{s t}^{2}+\mathrm{e}^{4 \phi / 3} d y^{2}
$$

between M-theory and IIA string frame backgrounds, one sees that the CSV [8] configuration

$$
d s_{s t}^{2}=-2 d y^{+} d y^{-}+\delta_{i j} d y^{i} d y^{j} \quad \quad \mathrm{e}^{2 \phi}=\mathrm{e}^{-3 y^{+}},
$$

Minkowski space with a linear dilaton, lifts to the M-theory plane wave metric

$$
d s_{11}^{2}=-2 d u d v+u \delta_{i j} d y^{i} d y^{j}+u^{-2}(d y)^{2},
$$

where $y^{+}=\log u$ (and $y^{-}=v$ ). We will now turn this around and consider the reduction of more general 11d plane wave metrics (in Rosen coordinates)

$$
d s_{11}^{2}=-2 d u d v+G_{i j}(u) d y^{i} d y^{j}+c(u) d y^{2}
$$

along $y$. Then one obtains the IIA string frame metric + null dilaton configuration

$$
\begin{aligned}
d s_{s t}^{2} & =c^{1 / 2}(u)\left(-2 d u d v+G_{i j}(u) d y^{i} d y^{j}\right) \\
\mathrm{e}^{2 \phi(u)} & =c(u)^{3 / 2} .
\end{aligned}
$$

The ten-dimensional string and Einstein frame metrics are also plane waves. In the string frame, the standard Rosen form is obtained by introducing the null coordinate (affine parameter) $y^{+}$,

$$
d y^{+}=c^{1 / 2}(u) d u,
$$


in terms of which (and $y^{-}=v$ ) the metric takes the standard Rosen form (A.1) with $g_{i j}\left(y^{+}\right)=$ $\left(c^{1 / 2} G_{i j}\right)\left(u\left(y^{+}\right)\right)$. The Einstein frame metric $d s_{e}^{2}=\exp (-\phi / 2) d s_{s t}^{2}$ is also manifestly a plane wave, written in the almost-Rosen form (A.2).

Let us now concentrate on the 11d singular homogeneous plane waves of the power-law form

$$
\begin{aligned}
d s_{11}^{2} & =-2 d u d v+\sum_{i} u^{2 n_{i}}\left(d y^{i}\right)^{2}+u^{2 b}(d y)^{2} \\
& =-2 d z^{+} d z^{-}+\sum_{a} \frac{n_{a}\left(n_{a}-1\right)}{\left(z^{+}\right)^{2}}\left(z^{a}\right)^{2}\left(d z^{+}\right)^{2}+\frac{b(b-1)}{\left(z^{+}\right)^{2}} z^{2}\left(d z^{+}\right)^{2}+\sum_{a}\left(d z^{a}\right)^{2}+(d z)^{2}
\end{aligned}
$$

The relation $d y^{+}=u^{b} d u$ (B.6) integrates to $y^{+}=u^{b+1} /(b+1)$ for $b \neq-1$ and to $y^{+}=\log u$ for $b=-1$. Thus the dilaton behaves as

$$
\mathrm{e}^{2 \phi(u)}=u^{3 b}=\left\{\begin{array}{cc}
{\left[(b+1) y^{+}\right]^{3 b /(b+1)}} & b \neq-1 \\
\mathrm{e}^{3 b y^{+}}=\mathrm{e}^{-3 y^{+}} & b=-1
\end{array}\right.
$$

While in general one obviously always finds a null dilaton in 10 dimensions, a linear dilaton, as in the CSV model (B.2), arises only for the special value $b=-1$ of the parameter $b$. By a suitable scaling of the coordinates, one can put the $b \neq-1$ IIA backgrounds into the normalised form (A.12,A.13)

$$
\begin{aligned}
d s_{s t}^{2} & =-2 d y^{+} d y^{-}+\sum_{i}\left(y^{+}\right)^{2 m_{i}}\left(d y^{i}\right)^{2} \\
& =-2 d z^{+} d z^{-}+\sum_{a} \frac{m_{a}\left(m_{a}-1\right)}{\left(z^{+}\right)^{2}}\left(z^{a}\right)^{2}\left(d z^{+}\right)^{2}+\sum_{a}\left(d z^{a}\right)^{2} \\
\mathrm{e}^{2 \phi} & =\left(y^{+}\right)^{3 b /(b+1)}
\end{aligned}
$$

with

$$
2 m_{i}=\frac{2 n_{i}+b}{b+1}
$$

For $b=-1$, on the other hand, one has

$$
\begin{aligned}
d s_{s t}^{2} & =-2 d y^{+} d y^{-}+\sum_{i} e^{\left(2 n_{i}-1\right) y^{+}}\left(d y^{i}\right)^{2} \\
& =-2 d z^{+} d z^{-}+\sum_{a}\left(2 n_{a}-1\right)^{2}\left(z^{a}\right)^{2}\left(d z^{+}\right)^{2}+\sum_{a}\left(d z^{a}\right)^{2}
\end{aligned}
$$

with a linear dilaton. This has the standard form of a metric of a non-singular symmetric plane wave (constant $A_{a b}$ ).

\section{B.2 Equations of Motion}

The 11d vacuum Einstein equations for the singular homogeneous plane wave (B.7) reduce to the algebraic condition

$$
\sum_{a} n_{a}\left(n_{a}-1\right)+b(b-1)=0
$$


In terms of the IIA parameters $m_{i}$ (B.10) for $b \neq-1$, this equation can be written as

$$
\sum_{i} m_{i}\left(m_{i}-1\right)=-\frac{3 b}{b+1}
$$

which implies

$$
\frac{3 b}{b+1} \leq 2
$$

The algebraic constraint (B.13) can be recognised as the Einstein-dilaton equation

$$
R_{++}\left(z^{+}\right)=-2 \partial_{+} \partial_{+} \phi\left(z^{+}\right)
$$

for the dilaton

$$
\phi\left(z^{+}\right)=\frac{3 b}{2(b+1)} \log z^{+} .
$$

Since all terms in (B.12) are positive when the parameters are sufficiently large (positive or negative), this strongly constrains their allowed range. A useful way of writing this equation is

$$
(b-2)(b+1)=-\sum_{a}\left(n_{a}-1 / 2\right)^{2} .
$$

Since the right hand side is non-positive, this leads to the constraint

$$
-1 \leq b \leq 2
$$

Thus the linear dilaton case $b=-1$ lies at the boundary of the allowed parameter range, and the only solution with $b=-1$ is the (lifted) CSV solution (B.3) with $n_{a}=1 / 2$. The solution with $b=2$ is, in a sense dual to the CSV background (the $11 \mathrm{~d}$ metrics with $b=-1$ and $b=2$ are isometric, but the reduction to $10 \mathrm{~d}$ is performed either along $\partial_{y}$ or along the dual isometry direction).

\section{B.3 Singularity Structure and Behaviour of the Dilaton at the Singularity}

We now want to analyse the behaviour of the dilaton at the singularity of the plane wave metrics for $b \neq-1$, and begin with a brief review of the situation for the $b=-1$ CSV background. As a symmetric plane wave, the $b=-1$ metric (B.11) is completely non-singular. It is isometric to the flat metric iff $2 n_{a}-1=0$. This is precisely the CSV background (B.2,B.3), and the only solution to the vacuum Einstein equations for $b=-1$.

Even though the string frame metric is flat, the IIA background as a whole should be considered to be singular [8], either because of the dilaton $\sim \exp -3 y^{+}$, which is singular as $y^{+} \rightarrow-\infty$, or because of the behaviour of the metric in the Einstein frame. This is compatible with the fact that the M-theory lift (B.3) of the CSV background is itself a singular homogeneous plane wave with a singularity at $u=0\left(y^{+}=\log u\right)$. Thus the singularity arises at strong string coupling and therefore, in the matrix string setting, at weak gauge coupling. Far from the singularity, on the other hand, the string coupling goes to zero. Since $y^{+}$can be identified with the relevant affine geodesic parameter, the singularity is located at infinite geodesic distance in the string frame metric. This is in contrast to what happens for the 11-dimensional lift of the CSV metric 
(B.3) (the singularity occurs at the finite value $u=0$ of the affine parameter $u$ ) or in the Einstein frame.

The $b \neq-1$ metrics (B.9) have a singularity at $y^{+}=0$ unless the metric is isometric to the flat metric, which is the case iff $m_{a}\left(m_{a}-1\right)=0$, i.e. $m_{a}=0$ or $m_{a}=1$, requiring also a constant dilaton $b=0$. The relation between the 11-dimensional and 10-dimensional string frame affine paramters $u$ and $y^{+}$is

$$
u \sim\left(y^{+}\right)^{1 /(b+1)}
$$

and the dilaton is

$$
\mathrm{e}^{2 \phi} \sim\left(y^{+}\right)^{3 b /(b+1)}
$$

Thus for $b+1>0$ (B.18), a condition implied by the Einstein equations, $u \rightarrow 0$ corresponds to $y^{+} \rightarrow 0$, and thus the singularity is always at finite geodesic distance, even in the string frame, in contrast to what happens in the CSV background.

Since $b+1>0$, it is evident that the behaviour of the dilaton at the singularity is determined by the sign of $b$,

$$
\begin{array}{cc}
\text { strong coupling singularity: } & -1<b<0 \\
\text { weak coupling singularity: } & 0<b \leq 2 .
\end{array}
$$

Finally, note that in the strongly coupled range the string coupling goes to zero at infinity, i.e. as $z^{+} \rightarrow \infty$, just as in the CSV model. In the weakly coupled range, on the other hand, the string coupling would blow up there. This can be remedied by noting [21] that the general solution of the IIA Einstein-dilaton equations (B.15), also includes a linear term in the dilaton solution (B.16),

$$
\phi\left(z^{+}\right)=\frac{3 b}{2(b+1)} \log z^{+}-c z^{+} .
$$

For $c>0$ and $b>0$ this has the effect that the string coupling now tends to zero both at the singularity $z^{+}=0$ and for $z^{+} \rightarrow \infty$. This is the case analysed from a string theory point of view in [21]. Since the metric-dilaton background for $c \neq 0$ does not arise from (or lift to) a singular homogeneous plane wave in 11 dimensions, and since, in the spirit of [23, 24], we regard the singular homogeneous plane wave metrics not as genuine comsological toy-models but as a near-singularity approximation of a singular space-time (in particular, we do not trust / take seriously the metric as $z^{+} \rightarrow \infty$ ), we will only consider the solutions with $c=0$.

\section{REFERENCES}

[1] M. Berkooz, D. Reichmann, A Short Review of Time Dependent Solutions and Space-like Singularities in String Theory, arXiv:0705.2146v1 [hep-th].

[2] T. Banks, W. Fischler, S. Shenker and L. Susskind, M theory as a matrix model: A Conjecture, Phys. Rev. D55 (1997) 5112, arXiv:hep-th/9610043.

[3] L. Susskind, Another conjecture about M(atrix) theory, arXiv:hep-th/9704080.

[4] L. Motl, Proposals on nonperturbative superstring interactions, arXiv:hep-th/9701025; T.Banks and N. Seiberg, Strings from matrices, Nucl. Phys. B497 (1997) 41, arXiv:hep-th/9702187. 
[5] R. Dijkgraaf, E.P. Verlinde and H.L. Verlinde, Matrix string theory, Nucl. Phys. B500 (1997) 43, arXiv:hep-th/9703030.

[6] W. Taylor, M. van Raamsdonk, Multiple D0-branes in Weakly Curved Backgrounds, Nucl.Phys. B558 (1999) 63-95, arXiv:hep-th/9904095v2; W. Taylor, M. van Raamsdonk, Multiple Dp-branes in Weak Background Fields, Nucl.Phys. B573 (2000) 703-734, arXiv:hep-th/9910052v1.

[7] R. Schiappa, Matrix Strings in Weakly Curved Background Fields, Nucl.Phys. B608 (2001) 3-50, arXiv:hep-th/0005145v2.

[8] B. Craps, S. Sethi and E.P. Verlinde, A Matrix Big Bang, JHEP 0510:005, (2005), arXiv:hep-th/0506180.

[9] B. Craps, A. Rajaraman and S. Sethi, Effective Dynamics of the Matrix Big Bang, Phys. Rev. D73 (2006) 106005, arXiv:hep-th/0601062.

[10] B. Craps, Big Bang Models in String Theory, Class. Quant. Grav. 23 (2006) S849, arXiv:hep-th/0605199.

[11] D. Robbins and S. Sethi, A Matrix Model for the Null-Brane, JHEP 0602 (2006) 052, arXiv:hep-th/0509204.

[12] E. Martinec, D. Robbins and S. Sethi, Toward the End of Time, JHEP 0608 (2006) 025, arXiv:hep-th/0603104.

[13] J. Bedford, C. Papageorgakis, D. Rodriguez-Gomez and J. Ward, Matrix Big Brunch, arXiv:hep-th/0702093.

[14] M. Li, A Class of Cosmological Matrix Models, Phys. Lett. B626 (2005) 202, arXiv:hep-th/0506260; M. Li and W. Song, Shock Waves and Cosmological Matrix Models, JHEP 0510 (2005) 073, arXiv:hep-th/0507185.

[15] B. Chen, The Time-dependent Supersymmetric Configurations in M-theory and Matrix Models, Phys. Lett. B632 (2006) 393, arXiv:hep-th/0508191; Hong-Zhi Chen and Bin Chen, Matrix Model in a Class of Time Dependent Supersymmetric Backgrounds, Phys. Lett. B638 (2006) 74, arXiv:hep-th/0603147.

[16] S.R. Das and J. Michelson, pp wave big bangs: Matrix strings and shrinking fuzzy spheres, Phys. Rev. D72 (2005) 086005, arXiv:hep-th/0508068; S.R. Das and J. Michelson, Matrix membrane big bangs and D-brane production, Phys. Rev. D73 (2006) 126006, arXiv:hep-th/0602099.

[17] T. Ishino and N. Ohta, Matrix String Description of Cosmic Singularities in a Class of Timedependent Solutions, Phys. Lett. B638 (2006) 105, arXiv:hep-th/0603215.

[18] N. Seiberg, Why is the Matrix Model Correct?, Phys. Rev. Lett. 79 (1997) 3577, arXiv:hep-th/9710009.

[19] A. Sen, D0 Branes on $T^{n}$ and Matrix Theory, Adv. Theor. Math. Phys. 2 (1998) 51, arXiv:hep-th/9709220.

[20] A. Sen, An Introduction to Non-perturbative String Theory, arXiv:hep-th/9802051.

[21] G. Papadopoulos, J.G. Russo and A.A. Tseytlin, Solvable model of strings in a time dependent plane wave background, Class. Quant. Grav. 20 (2003) 969, arXiv:hep-th/0211289.

[22] M. Blau, M. O’Loughlin, Homogeneous Plane Waves, Nucl. Phys. B654 (2003) 135-176, arXiv:hep-th/0212135.

[23] M. Blau, M. Borunda, M. O’Loughlin, G. Papadopoulos, Penrose Limits and Spacetime Singularities, Class. Quant. Grav. 21 (2004) L43-L49, arXiv:hep-th/0312029. 
[24] M. Blau, M. Borunda, M. O'Loughlin, G. Papadopoulos, The universality of Penrose limits near space-time singularities, JHEP 0407 (2004) 068, arXiv:hep-th/0403252

[25] G. Papadopoulos, Power-law singularities in string theory and M-theory, Class. Quant. Grav. 21 (2004) 5097-5120, arXiv:hep-th/0404172.

[26] P. Szekeres and V. Iyer, Spherically symmetric singularities and strong cosmic censorship, Phys. Rev. D47 (1993) 4362.

[27] M. Blau, D. Frank, S. Weiss, Fermi Coordinates and Penrose Limits, Class. Quantum Grav. 23 (2006) 3993-4010, arXiv:hep-th/0603109.

[28] M. Blau, S. Weiss, Penrose Limits vs String Expansions, Class. Quantum Grav. 25 (2008) 125014, arXiv:0710.3480v1 [hep-th]

[29] M. Blau and M. O'Loughlin, Multiple M2-Branes and Plane Waves, arXiv:0806.3253 [hep-th].

[30] J. Bagger and N. Lambert, Modeling Multiple M2's, Phys. Rev. D75 (2007) 045020, arXiv:hep-th/0611108; J. Bagger and N. Lambert, Gauge symmetry and supersymmetry of multiple M2-branes, Phys. Rev. D77 (2008) 065008, arXiv:0711.0955 [hep-th]; J. Bagger and N. Lambert, Comments on multiple M2-branes, JHEP 0802 (2008) 105, arXiv:0712.3738 [hep-th].

[31] A. Gustavsson, Algebraic structures on parallel M2-branes, arXiv:0709.1260 [hep-th]; A. Gustavsson, Selfdual strings and loop space Nahm equations, JHEP 0804 (2008) 083, arXiv:0802.3456 [hep-th].

[32] J. Gomis, G. Milanesi and J.G. Russo, Bagger-Lambert Theory for General Lie Algebras, arXiv:0805.1012 [hep-th]; S. Benvenuti, D. Rodriguez-Gomez, E. Tonni and H. Verlinde, $N=8$ superconformal gauge theories and M2 branes, arXiv:0805.1087 [hep-th]; Pei-Ming Ho, Y. Imamura and Y. Matsuo, M2 to D2 revisited, arXiv:0805.1202 [hep-th].

[33] D. Frank and S. Weiss, to appear.

[34] M. Douglas, D. Kabat, P. Pouliot, S.Shenker, D-branes and Short Distances in String Theory, Nucl.Phys. B485 (1997) 85-127, arXiv:hep-th/9608024v2.

[35] G. Lifshytz, DLCQ-M(atrix) Description of String Theory, and Supergravity, Nucl. Phys. B534 (1998) 83, arXiv:hep-th/9803191.

[36] R. Penrose, Any space-time has a plane wave as a limit, in Differential geometry and relativity, Reidel, Dordrecht (1976) pp. 271-275.

[37] R. Gueven, Plane wave limits and T-duality, Phys. Lett. B482 (2000) 255-263, arXiv:hep-th/0005061.

[38] M. Blau, J. Figueroa-O'Farrill, G. Papadopoulos, Penrose limits, supergravity and brane dynamics, Class. Quant. Grav. 19 (2002) 4753, arXiv:hep-th/0202111.

[39] A. Shomer, Penrose limit and DLCQ of string theory, Phys. Rev. D68 (2003) 086002, arXiv:hep-th/0303055.

[40] M.M. Sheikh-Jabbari, Tiny Graviton Matrix Theory: DLCQ of IIB Plane-Wave String Theory, A Conjecture, JHEP 0409 (2004) 017, arXiv:hep-th/0406214.

[41] M. Torabian, Matrix Theory for the DLCQ of Type IIB String Theory on the AdS/Plane-wave, Phys. Rev. D76 (2007) 026006, arXiv:hep-th/0701046.

[42] D. Marolf, L. Martucci and P.J. Silva, Actions and Fermionic symmetries for D-branes in bosonic backgrounds, JHEP 0307 (2003) 019, arXiv:hep-th/0306066.

[43] G.J. Galloway, Cosmological Spacetimes with $\Lambda>0$, arXiv:gr-qc/0407100. 
[44] B. McInnes, Inaccessible Singularities in Toral Cosmology, Class. Quant. Grav. 24 (2007) 1605, arXiv:gr-qc/0611101.

[45] B. Craps, T. Hertog, N. Turok, Quantum Resolution of Cosmological Singularities using AdS/CFT, arXiv:0712.4180v2 [hep-th].

[46] A. Awad, S. Das, S. Nampuri, K. Narayan, S. Trivedi, Gauge Theories with Time Dependent Couplings and their Cosmological Duals, arXiv:0807.1517v2 [hep-th]. 